\title{
Optimal quantum measurement of finite-dimensional systems and coherent anti-Stokes Raman spectroscopy
}

\author{
GAO Fang ${ }^{1,2}$, WANG YaoXiong ${ }^{1} \&$ SHUANG Feng ${ }^{1 *}$ \\ ${ }^{1}$ Institute of Intelligent Machines, Chinese Academy of Sciences, Hefei 230031, China; \\ ${ }^{2}$ State Key Laboratory of Robotics, Shenyang Institute of Automation, Chinese Academy of Sciences, Shenyang 110016, China \\ Received November 21, 2011; accepted February 3, 2012; published online May 4, 2012
}

\begin{abstract}
Quantum measurement is a fundamental problem in quantum control theory and experiments. It can obtain unknown information of quantum systems, and can also change state of the systems inevitably. Both the outcome and back action could be used to control quantum systems. This paper presents recent research progress about optimal control of state transformation in finite-dimensional quantum systems by back action of non-selective quantum measurement, and optimal control of signal and background of CARS (coherent anti-Stokes Raman spectroscopy) by phase shaping technique. In measurement sequence control of finite-dimensional quantum systems, the necessary condition for critical points of the underlying state transformation objective is found to be a highly symmetric form as a chain of equalities, and analytical and numerical solutions in several cases are explored. In the CARS control, it is found that the maximal resonant signal and minimal background at a specific frequency can be achieved by shaping the probe pulse only while keeping pump and Stokes pulses in transform limited forms (TLFs). An arctan-type phase function is obtained for the probe pulse to simultaneously enhance the resonant signal and suppress the background. For broadband background elimination, we find that the optimal phase shaping scheme of probe pulse is quasi-time-delay while keeping the pump and Stokes pulses in TLFs. These conclusions could help design control strategies of quantum devices.
\end{abstract}

quantum measurement, finite-dimensional system, coherent anti-Stokes Raman spectroscopy, phase shaping

Citation: Gao F, Wang Y X, Shuang F. Optimal quantum measurement of finite-dimensional systems and coherent anti-Stokes Raman spectroscopy. Chin Sci Bull, 2012, 57: 2215-2222, doi: 10.1007/s11434-012-5182-3

Quantum measurement is a fundamental problem in quantum theories and has been well discussed since the born of quantum mechanics. Dirac and von Neumann proposed for the first time that the quantum system should collapse from the initial mixed state to one eigenstate upon measurement $[1,2]$. Then in the $1950 \mathrm{~s}$, physicists explored beyond this collapse theory from different perspectives, such as the hidden variable theory by Bohm [3,4] and the many-worlds interpretation by Everett [5]. After 1970s, theorists tried to develop quantum measurement theory under the framework of the evolution of Schrödinger equation, for example, Zeh argued that the probability interpretation is compatible with an objective interpretation of the wave function [6] and Zurek

*Corresponding author (email: fshuang@iim.ac.cn) proposed the decoherence interpretation which emphasizes the entanglement between the equipment and environment [7]. Different from the measurement in classical physics, quantum measurement are not independent of the observed system. Instead, the measurement itself will have back-action effect, which may be employed to control the quantum system. Quantum measurement can be divided into selective and non-selective measurements [8]. The former has been widely studied for purified quantum states. Quantum Zeno and antiZeno effects [9] are two extreme examples induced by nonselective measurement, which can be employed for quantum control in ideal experimental conditions. However, how to achieve the target state in real situations by measurementinduced control is still an open question. Significant research efforts have been put forth to make progress in this field. We 
have previously studied the optimal projective measurements $[10,11]$ in a simple two-level quantum system, and shown that quantum measurements can cooperate with lasers to control the system more effectively [12].

In quantum world, information acquisition is often achieved by indirect measurement, such as the spectra induced by the interaction of the system and lasers. Since the discovery of Raman scattering, Raman spectra have been an important mapping tool of molecular vibrations. Combined with microscopy techniques, Raman spectra can also be employed to carry out a three-dimensional visual study of biological samples. However, normal Raman signals are too weak and easily merged into background fluorescence. These disadvantages disappear in CARS. As a four-wave nonlinear process, CARS [13] has been widely used in the past few decades for studying chemical systems in solutions, reactions in gas phases, and vibrational dynamics in gas and condensed phases. CARS microscopy is a recently implemented technique for imaging of biological species, which is pioneered by Duncan et al. using two dye lasers and developed [14] by Xie et al. with a more advanced system with high sensitivity $[15,16]$. As a combination of ultrafast nonlinear spectroscopy and microscopy, CARS microscopy is a high chemically selective and sensitive microscopy technique that uses CARS signal of the unlabeled sample, and it provides higher spatial resolution than two-photon fluorescence microscopy. In CARS microscopy, mostly only one vibrational mode (e.g. $\mathrm{CH}$ stretch motion) is probed using picosecond pulses, which can produce sharp signal and low background $[15,17,18]$. However, people usually have to detect multiple modes in experiments to distinguish large molecules such as protein. In this case, it is necessary to bring in broadband femtosecond pulses. However, there emerges a dilemma that when the broadband femtosecond pulses are employed, the nonresonant background may submerge the resonant signal and the fine vibrational structure of CARS $[19,20]$. Theoretical and experimental studies have demonstrated that the disadvantage can be effectively avoided by coherence control. The background suppression without loss of the resonant signal via shaping pulses has been explored widely [20,21], which benefits from the development of phase shaping techniques for femtosecond pulses. For example, Silberberg et al. combined the phase and polarization control to yield backgroundfree single-pulse multiplex CARS spectra with a high spectral resolution [22].

This work presents recent study on optimal state control of finite-dimensional quantum systems by back action from proper quantum measrement, and optimal control of signal and background of CARS by phase shaping. Control strategies are explored analytically or numerically. The paper is organized as follows: Section 1 takes finite-dimensional quantum systems as example to present the corresponding measurement-induced control strategies. Section 2 shows optimal phase shaping schemes for CARS in different cases. Section 3 gives the concluding remarks and some open ques- tions to answer in the future.

\section{Measurement-induced control}

In this section, we first review some prior works about measurement-assistant control and introduce quantum system control which employs measurement only. Then, some analytical results about two-level systems are presented. For a general finite-dimensional quantum system, the necessary condition for a measurement sequence to be a critical point is obtained, and some analytical and numerical solutions in several cases are explored based on this condition.

\subsection{Measurement in quantum control}

Most studies of quantum control focus on coherent manipulation of quantum systems, since coherent manipulation, even reversible manipulation, is more powerful than incoherent process. Due to great difficulty of coherent control of quantum systems in laboratory experiments, other incoherent controls, such as laser noise, decoherence from the environment, and quantum observations, have drawn attention. In contrast to the natural expectation that incoherent forces will introduce deleterious effects toward achieving desired control [23], recent studies [24,25] have shown that controlled quantum dynamics can survive intense field noise and decoherence, and even cooperate with them under special circumstances [26]. For example, a suitably optimized environment, as a incoherent control force, was suggested as a supplement to coherent control to provide a general tool for selective manipulation of both the Hamiltonian and dissipative aspects of the system dynamics $[27,28]$. Optimal coherent control fields were shown to be capable of cooperating or fighting with quantum measurements, and the performance may be optimized to achieve more effective control of the quantum dynamics.

Quantum measurement, which serves as an incoherent driving force, could also be used to manipulate the quantum dynamics. It is well-known that both the outcome and backaction of quantum measurements can be employed to control quantum processes. In the standard closed-loop optimal control experiments [29], the recorded outcome, which comes from a non-selectively measurement of a system at the end of a trial control, is processed by a learning algorithm to further optimize the laser pulse. Quantum measurements were also applied to map an unknown mixed state to some target pure state [30] by exploiting the resultant back action based on which coherent control can be selectively performed [31], and shown to be necessary in some laser control [32], dephasing decoherence control [33] and incoherent control problems [34]. The effect of non optimized measurements upon control by lasers was investigated in $[35,36]$. Quantum measurements also make a quantum system more controllable by breaking dynamical symmetries, e.g. the quantum Zeno and 
anti-Zeno effects. In the absence of an active coherent control, instantaneous projective measurements were shown to be capable of driving population transfer in a two-level system, and a scheme for maximal population transfer was found under an arbitrary finite number of measurements $[10,11]$. Numerical simulations have been carried out to investigate the ability of measurements in quantum control [12].

Although both the outcome and back-action from measurements could be used to control quantum processes, we just consider the latter one, i.e. only non-selective measurement process is involved. In the subsections below, we will review some control schemes using measurements only and present some optimal control strategies.

\subsection{Some analytical results in measurement-induced quantum control}

We consider the quantum state control of a two-level system by projective measurements only and generalize some of results to general $M$-dimensional quantum systems. For an ideal measurement $M$ applies to a system characterized by a density matrix $\rho$, a non-selective measurement process is represented as $M(\rho)=\sum_{k} P_{k} \rho P_{k}$ in which $\left\{P_{k}\right\}$ are the projective operators which satisfy $P_{i} P_{j}=P_{i} \delta_{i j}$ and $\sum_{i} P_{i}=1$. The objective in this circumstance is to drive the quantum system from an initial state $\rho_{0}=|0\rangle\langle 0|$ to a final state $\rho_{f}=|1\rangle\langle 1|$. We define the target function $J$ as

$$
\operatorname{Tr}\left[\rho_{f} \cdot\left(M^{N} \cdots \cdot M^{2} \cdot M^{1}\left(\rho_{0}\right)\right)\right]
$$

where operation $M^{i}$ implies $i$-th measurement. Note that we do not consider the free evolution of the system in eq. (1), since it is easy to include it via a picture transformation $[10,11]$.

For two-level quantum systems, analytical solutions for population transfer by optimized instantaneous projective measurements is obtained $[10,11]$. In these cases, we can associate each measurement with two projectors, and find the optimal transfer population between the initial and final mixed states, $\rho_{i}$ and $\rho_{f}$. Assume that these two density matrices can be represented as $\rho_{i, f}=\frac{1}{2}\left(\mathbb{I}+\boldsymbol{a}_{i, f} \cdot \sigma\right)$ where $\sigma=\left(\sigma_{1}, \sigma_{2}, \sigma_{3}\right)$ is the vector of Pauli matrices. For some fixed measurement time, in the optimal yield is

$$
J_{N}^{\max }=\frac{1}{2}+\frac{1}{2}\left|\boldsymbol{a}_{i}\right|\left|\boldsymbol{a}_{f}\right|\left(\cos \frac{\Delta \varphi}{N+1}\right)^{N+1}
$$

where $\Delta \varphi$ is the angle between vectors $\boldsymbol{a}_{i}$ and $\boldsymbol{a}_{f}$. For pure orthogonal initial and final states, $\left|\boldsymbol{a}_{i}\right|$ and $\left|\boldsymbol{a}_{f}\right|$ are both equal to 1 and $\Delta \varphi$ equal to $\pi$. Consequently, eq. (2) will reduce to

$$
J_{N}^{\max }=\frac{1}{2}\left[1+\left(\cos \frac{\pi}{N+1}\right)^{N+1}\right] .
$$

Thus, with the help of Pauli matrices, all two-level measurement control cases for arbitrary initial and final states have been solved.
It is much complicated for a general $M$-dimensional system because the choice of the projectors in each measurement is not unique. Suppose $\left\{I_{1}, \cdots, I_{n}\right\}$ are diagonal matrices whose diagonal elements are either 1 or 0 . For $n$ projectors $\left\{P_{i}\right\}$ in each measurement, there exists a unitary matrix $U$ which can diagonalize all the projectors simultaneously, i.e. $P_{i}=U I_{i} U^{\dagger}$. The summation of all $I_{i}$ is the identity operator in the $M$-dimensional space:

$$
\sum_{i=1}^{n} I_{i}=U^{\dagger}\left(\sum_{i=1}^{n} P_{i}\right) U=I .
$$

Consequently, quantum measurements could be classified by the set of diagonal matrices $\left\{I_{1}, \cdots, I_{n}\right\}$, and a measurement $\mathcal{M}^{(k)}$ in a class can be characterized by a unitary matrix $U_{k}$, and the target function converts to

$$
J\left[U_{1}, \ldots, U_{m}\right]=\operatorname{Tr}\left[\theta \mathcal{M}\left(U_{m}\right) \ldots \mathcal{M}\left(U_{1}\right) \rho_{0}\right] .
$$

This is an optimized problem with unitary constraints. Treat these unitary matrices as variables and follow the most common approach by considering the first-order term in the Taylor expansion, we obtain a chain of equalities for $m$-times measurements:

$$
\begin{aligned}
& {\left[\rho_{0}, \mathcal{M}\left(U_{1}\right) \ldots \mathcal{M}\left(U_{m}\right) \theta\right] } \\
= & \ldots \\
= & {\left[\mathcal{M}\left(U_{k}\right) \ldots \mathcal{M}\left(U_{1}\right) \rho_{0}, \mathcal{M}\left(U_{k+1}\right) \ldots \mathcal{M}\left(U_{m}\right) \theta\right] } \\
= & \ldots \\
= & {\left[\mathcal{M}\left(U_{m}\right) \ldots \mathcal{M}\left(U_{1}\right) \rho_{0}, \theta\right], }
\end{aligned}
$$

which is the necessary condition for optimal measurements to yield a maximal outcome [37].

We present three analytical cases with single measurement below [37]. The first case is a direct generalization of twolevel systems. Consider the transformation between two pure states $|\alpha\rangle$ and $|\beta\rangle$ induced by single measurement represented by $M 1$-rank projectors. Single measurement means only one equation in eq. (6) is involved:

$$
[\mathcal{M}(U) \rho, \theta]=[\rho, \mathcal{M}(U) \theta],
$$

in which $\rho=|\alpha\rangle\langle\alpha|\theta=| \beta\rangle\langle\beta|$. In this situation, the maximal state transition probability between two pure states $|\alpha\rangle$ and $|\beta\rangle$ is

$$
J_{\max }=\frac{1+|\langle\alpha \mid \beta\rangle|^{2}}{2} .
$$

It is interesting to note that, the maximum of $M$-dimensional system is just the same as that of a simple two-level system spanned by the initial state $|\alpha\rangle$ and the final state $|\beta\rangle$, which has been extensively studied $[10,11]$.

Comparing with the first one, the second case involves only two projectors. One projector is rank 1, i.e. $P=|v\rangle\langle v|$ and the other is the complement $Q=I-P$. This measurement procedure produces only two exclusive results, success or failure, e.g. the test of an unknown state whether it has a certain component characterized by a pure state. Suppose that the desired pure desired state is $|1\rangle$ and the initial mixed 
state is $\rho$, then after the measurement, the initial state $\rho$ becomes $\rho^{\prime}=P \rho P+(I-P) \rho(I-P)$ and our goal is to maximize $\operatorname{Tr}\left(\rho^{\prime}|1\rangle\langle 1|\right)$. Further calculation shows that $J$ have the maximum as

$$
J_{\max }=\frac{\rho_{11}+\operatorname{Max}\left\{\lambda_{i}\right\}}{2},
$$

where $\left\{\lambda_{i}\right\}$ are the eigenvalues of $\rho$.

The third one corresponds to the transformation between two orthogonal mixed states $\rho$ and $\theta$, i.e. $\operatorname{tr}(\rho \theta)=0$. Under the same two-projector measurements assumption as the second case, we will find

$$
J_{\max }=\frac{1}{2} \rho_{\max } \theta_{\max }
$$

when the vector $|v\rangle$ in the projector $P=|v\rangle\langle v|$ is $|v\rangle=$ $\frac{1}{\sqrt{2}}\left(\left|\rho_{\max }\right\rangle+\left|\theta_{\max }\right\rangle\right)$. Here $\left|\rho_{\max }\right\rangle$ and $\left|\theta_{\max }\right\rangle$ are the eigenvectors of $\rho$ and $\theta$ corresponding to the largest eigenvalues, $\rho_{\max }$ and $\theta_{\max }$, respectively.

These three cases are all based on single measurement. For many times measurement with arbitrary initial and final states, we need to find a way to analytically solve eq. (7) with two arbitrary mixed states. This is intractable even for special diagonal mixed states and numerical approach is required to explore the properties of these cases.

\subsection{Numerical simulation aspects}

Since eq. (6) is not easy to solve for general initial and final states, we resort to numerical simulations. For a general $M$ dimensional system, when we consider the population transfer between two orthogonal pure states induced by measurements like two-level cases, numerical calculation implies that the optimal control scheme reduces to that of two-level cases dramatically. In other words, a series of measurements manipulate the states in the subspace spanned by the initial and final states only and other $M-2$ levels are negligible. This observation greatly simplifies the control process for multilevel cases.
Another numerical result is about the continuous measurements. In [11], two-level systems with continuous measurements are investigated, and a linear trial solution is proposed to optimize the final population. Although it has not been proved, this linear solution is strongly supposed to be the optimal one numerically.

\section{Phase shaping in CARS}

In this section, theoretical analysis of different phase shaping schemes in quantum control of CARS is presented with the aim of enhancing signal and suppressing background simultaneously. Phase shaping strategies at a specific frequency as well as for broadband are provided.

\subsection{Some notations}

As shown in Figure 1, CARS is a four-wave nonlinear process. Three laser pulses are used: the pump pulse $E_{p}\left(\omega_{p}\right)$, the Stokes pulse $E_{s}\left(\omega_{s}\right)$, and the probe pulse $E_{p r}\left(\omega_{p r}\right)$. The CARS signal is a coherent superposition of resonant nonlinear third order polarization $P_{r}^{(3)}\left(\omega_{a s}\right)$ and non-resonant nonlinear third order polarization $P_{n r}^{(3)}\left(\omega_{a s}\right)$ :

$$
I_{\mathrm{CARS}}\left(\omega_{a s}\right)=\left|P_{r}^{(3)}\left(\omega_{a s}\right)+P_{n r}^{(3)}\left(\omega_{a s}\right)\right|^{2},
$$

with

$$
\begin{aligned}
& P\left(\omega_{a s}\right)=C \iiint_{-\infty}^{+\infty} \mathrm{d} \omega_{p} \mathrm{~d} \omega_{s} \mathrm{~d} \omega_{p r} \frac{A\left(\omega_{p}, \omega_{s}, \omega_{p r}, \omega_{a s}\right)}{\Omega_{R}-\left(\omega_{p}-\omega_{s}\right)-i \Gamma}, \\
& P_{n r}^{(3)}\left(\omega_{a s}\right)=\chi_{n r} \iiint_{-\infty}^{+\infty} \mathrm{d} \omega_{p} \mathrm{~d} \omega_{s} \mathrm{~d} \omega_{p r} A\left(\omega_{p}, \omega_{s}, \omega_{p r}, \omega_{a s}\right) .
\end{aligned}
$$

Here $A\left(\omega_{p}, \omega_{s}, \omega_{p r}, \omega_{a s}\right)$ is equal to $E_{p}\left(\omega_{p}\right) E_{s}^{*}\left(\omega_{s}\right) E_{p r}\left(\omega_{p r}\right)$ $\times \delta\left(\omega_{a s}-\omega_{p}+\omega_{s}-\omega_{p r}\right), \Omega_{R}$ is the Raman frequency between

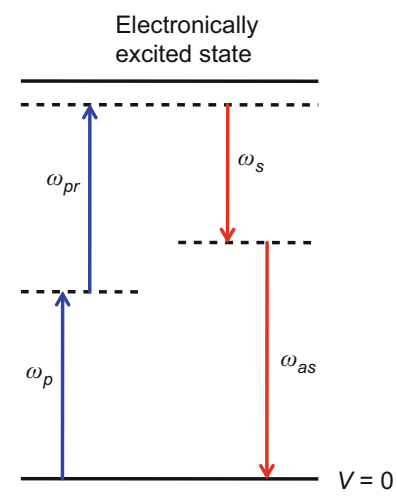

(c) Twp-photon enhancement nonresonant contribution

Figure 1 Energy level diagram of the CARS process. (a) Resonant signal generation: pump and Stokes pulses generate coherence between two vibrational levels, when they have a frequency difference which coincides with the Raman resonance $\Omega_{R}$. Henceforth the following probe pulse induces the anti-Stokes signal. (b) Non-resonant background contribution 1: one part of the non-resonant background is produced via the intermediate virtual state that does not reflect the resonant molecular energy level. (c) Non resonant background contribution 2: the other part of the non-resonant contribution origins from the two-photon enhancement process. 
energy levels 1 and 2, $2 \Gamma$ is the level width, $C$ is a constant which depends on the material property, and $\chi_{n r}$ is the nonresonant third-order susceptibility.

In our paper, $\Omega_{p}, \Omega_{s}$ and $\Omega_{p r}$ are defined as the carrier frequency of pump, Stokes and probe pulse, respectively. If all the pulses are transform limited pulses, then the peaks of resonant and non-resonant signal in the spectrum both locate at the frequency $\Omega_{a s}=\Omega_{p}-\Omega_{s}+\Omega_{p r}$.

The Gaussian amplitude profile in frequency domain is used for pump, Stokes and probe pulses in all theoretical treatments and simulations:

$$
E_{i}(\omega)=\frac{E_{i}}{\Delta_{i}^{1 / 2}} e^{-\left(\omega-\Omega_{i}\right)^{2} / \Delta_{i}^{2}} e^{i \Phi(\omega)}, i=\{P, S, P r\},
$$

where $\Phi(\omega)$ is the frequency-domain phase profile. For simplicity, a concise frequency variable translation, $\omega_{p r}=\omega_{p r^{\prime}}+$ $\Omega_{p r}$, is made (so $\Phi_{p r}\left(\omega_{p r}\right)=\Phi_{p r}\left(\omega_{p r^{\prime}}+\Omega_{p r}\right)$ is written as $\Phi_{p r^{\prime}}\left(\omega_{p r^{\prime}}\right)$ in the following), and we write $P_{r}^{(3)}\left(\Omega_{a s}\right)$ as $P_{r}$. In addition, $\Delta_{p}=\Delta_{p r}=\Delta_{s}=\Delta$ is assumed in the following.

\subsection{Signal peak enhancement and non-resonant back- ground local suppression}

In this subsection, control strategies to maximize the resonant signal enhancement or eliminate the background at $\Omega_{a s}$ are presented.

When only the probe pulse is shaped, the resonant signal equals to $C \sqrt{\frac{\pi}{2 \Delta}} \int_{-\infty}^{\infty} \frac{\mathrm{d} \omega_{p r^{\prime}}}{\sqrt{\omega_{p r^{\prime}}^{2}+\Gamma^{2}}} \exp \left[-\frac{3 \omega_{p r^{\prime}}^{2}}{2 \Delta^{2}}\right] \exp \left[i\left(\Phi_{p r^{\prime}}\left(\omega_{p r^{\prime}}\right)\right.\right.$ $\left.\left.+\alpha\left(\omega_{p r^{\prime}}\right)\right)\right]$, where $\alpha\left(\omega_{p r^{\prime}}\right)=-\arctan \left(\omega_{p r^{\prime}} / \Gamma\right)+\pi / 2$ is confined in the domain $[0, \pi]$. Then it is easily to see that $\left|P_{r}\right|^{2}$ reaches its maximal value when the probe pulse takes the phase function $\Phi_{p r^{\prime}}\left(\omega_{p r^{\prime}}\right)=\arctan \left(\omega_{p r^{\prime}} / \Gamma\right) . \quad\left|P_{n r}\left(\Omega_{a s}\right)\right|^{2}$ can also reach its minimal value 0 with probe-only shaping scheme, that is, when $\exp \left[i \Phi_{p r}\left(\omega_{p r^{\prime}}\right)\right]$ is an asymmetrical function (e.g. $\Phi_{p r}\left(\omega_{p r^{\prime}}\right)$ is the $\pi$ step phase function). However, the $\pi$ step phase function could only eliminate local component of background around $\omega_{a s}=\Omega_{a s}$ and form a dip in the spectrum. Thus the optimal phase function obtained by optimizing $\left|P_{n r}\left(\Omega_{a s}\right)\right|^{2}$ is locally optimal for the whole spectrum, which means that the background may still affect the resonant signal away from the position of $\omega_{a s}=\Omega_{a s}$ in the spectrum. Hence, the broadband background suppression method is necessary for CARS, which will be discussed in subsection 2.4 .

In experiments, there are several configurations of CARS: three-pulse CARS, two-pulse CARS (the pump pulse acts as probe pulse in the experiment) and single-pulse CARS [21] (all three photons required are supplied by the same short optical pulse). We will see in the following if three-pulse CARS could achieve better performance than the probe-only shaping scheme in the resonant signal enhancement since this configuration has the most control freedoms.

From eq. (12),

$P_{r}^{(3)}\left(\Omega_{a s}\right)=\frac{1}{\Delta^{3 / 2}} \int_{-\infty}^{\infty} \frac{C}{\omega_{p r^{\prime}}-i \Gamma} e^{-\frac{3 \omega_{p r^{\prime}}^{2}}{2 \Delta^{2}}+i \Phi_{p r^{\prime}}\left(\omega_{p r^{\prime}}\right)}$

$$
\begin{aligned}
& \times \int_{-\infty}^{\infty} e^{-\frac{2\left(\omega_{p^{\prime}}+\omega_{p r^{\prime}} / 2\right)^{2}}{\Delta^{2}}} e^{i \Phi_{p^{\prime}}\left(\omega_{p^{\prime}}\right)-i \Phi_{s^{\prime}}\left(\omega_{p^{\prime}}+\omega_{p r^{\prime}}\right)} \mathrm{d} \omega_{p r^{\prime}} \\
\leqslant & \left|\frac{1}{\Delta^{3 / 2}} \int_{-\infty}^{\infty}\right| \frac{C}{\omega_{p r^{\prime}}-i \Gamma} e^{-\frac{3 \omega_{p p^{\prime}}^{2}}{2 \Delta^{2}}+i \Phi_{p r^{\prime}}\left(\omega_{p r^{\prime}}\right)}\left|\cdot \sqrt{\frac{\pi}{2}} \Delta \cdot \mathrm{d} \omega_{p r^{\prime}}\right| \\
\leqslant & C \sqrt{\frac{\pi}{2 \Delta}} e^{\frac{3 \Gamma^{2}}{4 \Delta}} \operatorname{BesselK}\left(0, \frac{3 \Gamma^{2}}{4 \Delta^{2}}\right) .
\end{aligned}
$$

The first equality holds when $\Phi_{p^{\prime}}$ and $\Phi_{s^{\prime}}$ is constant, and the second when $\Phi_{p r^{\prime}}\left(\omega_{p r^{\prime}}\right)=\arctan \left(\omega_{p r^{\prime}} / \Gamma\right)$. Hence the maximal resonant signal is only achieved when the pump and Stoke pulse are unshaped transform limited pulses, and the probe pulse takes phase function $\Phi_{p r^{\prime}}\left(\omega_{p r^{\prime}}\right)=\arctan \left(\omega_{p r^{\prime}} / \Gamma\right)$. This fact means that any two-pulse or three-pulse shaping scheme is not necessary for optimizing resonant signal, because the simple probe-only shaping scheme could get optimal resonant signal. This conclusion still holds when $\Delta_{p}, \Delta_{p r}$ and $\Delta_{s}$ are different.

\subsection{Simultaneous resonant signal peak enhancement and non-resonant background local suppression}

In last subsection, the optimization of resonant signal and non-resonant background of the CARS process is treated separately, but in real experiments, the signal and background are always being detected together. Thus it is necessary to study the balance between the resonant signal enhancement and non-resonant background suppression, which is a multiobjective optimization problem. In this subsection, we will consider how to enhance the resonant signal while suppressing the background at a specific frequency by shaping the probe pulse.

The weighted difference of resonant signal and nonresonant background intensities is chosen as the objective functional:

$$
J=\left|P_{r}\right|^{2}-k\left|P_{n r}\right|^{2},
$$

where $k$ is the weight factor. By maximizing the intensity difference, we can achieve a large signal-background-ratio with the precondition of a considerable resonant signal intensity. This objective functional $J$ is the balance of the minimization of $\left|P_{n r}\right|^{2}$ and maximization of $\left|P_{r}\right|^{2}$.

The CMA-ES algorithm [38, 39], which is reliable for global optimization especially for the phase shaping scheme, is used here as the numerical optimization method. Using parameters $C=1$ and $\chi_{n r}=0.1$, the corresponding numerical optimal phases are shown in Figure 2. According to the variation condition $\delta J / \delta \Phi_{p r^{\prime}}\left(\omega_{p r^{\prime}}\right)=0$, we can get the solution for maximal value of $J$, and the optimal phase is a modified arctan-type function:

$$
\begin{aligned}
& \Phi_{p r^{\prime}}\left(\omega_{p r^{\prime}}\right)=\arctan \left(\frac{\omega_{p r^{\prime}}}{\Gamma-k\left(\chi_{n r} / C\right)^{2} \gamma\left(\omega_{p r^{\prime}}^{2}+\Gamma^{2}\right)}\right)+\theta, \\
& \gamma=\frac{\left\{\int_{-\infty}^{\infty} e^{-\frac{3 x^{2}}{2 \Delta^{2}}} \cos \left(\arctan \left(\frac{x}{\Gamma-k\left(\chi_{n r} / C\right)^{2} \gamma\left(x^{2}+\Gamma^{2}\right)}\right)\right) \mathrm{d} x\right\}}{\left\{\int_{-\infty}^{\infty} e^{-\frac{3 x^{2}}{2 \Delta^{2}}} \frac{1}{\sqrt{x^{2}+\Gamma^{2}}} \sin \left(\arctan \left(\frac{x}{\Gamma-k\left(\chi_{n r} / C\right)^{2} \gamma\left(x^{2}+\Gamma^{2}\right)}\right)+\alpha(x)\right) \mathrm{d} x\right\}},
\end{aligned}
$$



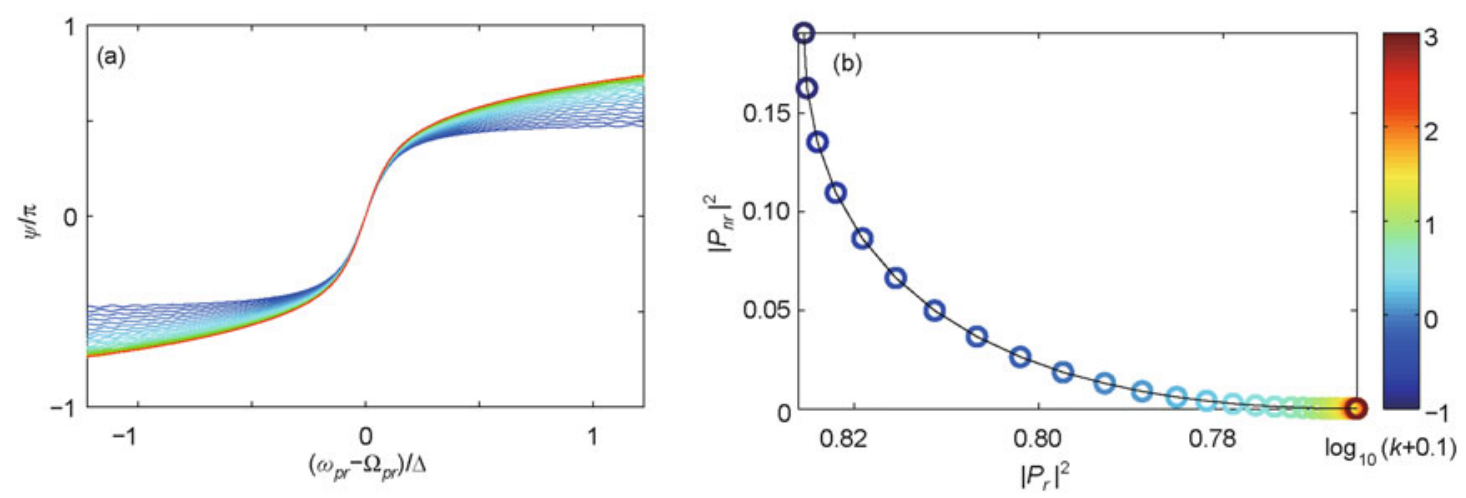

Figure 2 (a) Numerical optimal phase function of the probe pulse for $\left|P_{r}\right|^{2}-k\left|P_{n r}\right|^{2}$ with different weight $k$. All the phase functions in this figure could suppress the background to a small quantity. (b) The Pareto surface for the optimization of signal enhancement and background suppression. With different weight $k$, the value of $\left|P_{r}\right|^{2}$ is bounded in $[0.765,0.828]$, while $\left|P_{n r}\right|^{2}$ is always much smaller than $\left|P_{r}\right|^{2}$.

where $\theta$ is a trivial phase angle. The parameter $\gamma$ in (17) can be solved iteratively [40].

When the weight factor $k=0$, the optimization of $J$ is reduced to the maximization of resonant signal, and its solution $\arctan \left(\omega_{p r^{\prime}} / \Gamma\right)$ is also consistent with the result in subsection 2.2. The practical choice of $k$ depends on the ratio of $\chi_{n r} / C$. When $\chi_{n r} / C$ is large (large background), it is better to choose a large value of $k$, which will give a solution close to the limit point on the right side of the Pareto surface. When $\chi_{n r} / C$ is small, a small $k$ is appropriate. When $\chi_{n r} / C$ approaches zero, $k=0$ is the best choice, in which case it is no need to consider a very small background.

\subsection{Phase shaping for broadband}

We have studied the resonant signal enhancement and nonresonant background suppression at a specific frequency. However, sometimes in experiments, people need a large resonant signal and low non-resonant background in the whole spectrum, so the broadband non-resonant background suppression is necessary. An appropriate choice of the objective functional is the integration in the spectrum:

$$
J\left[\Phi_{p r}, \Phi_{p}, \Phi_{s}\right]=I_{r}-I_{n r},
$$

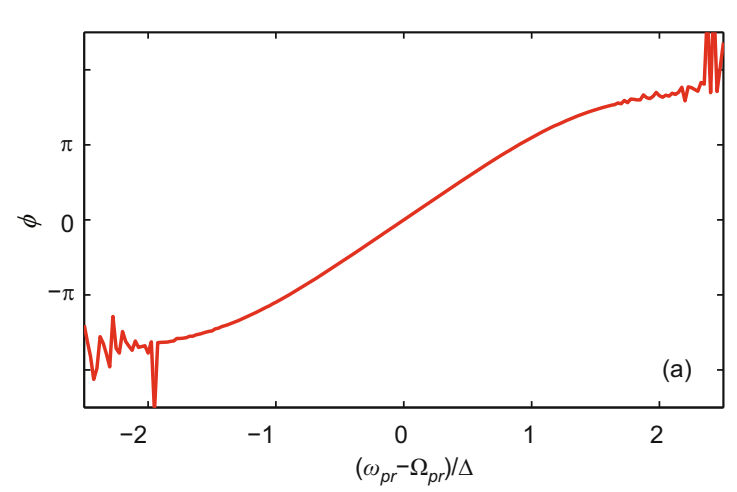

$$
\begin{aligned}
& I_{r}=\int_{-\infty}^{+\infty}\left|P_{r}\left(\omega_{a s}\right)\right|^{2} \mathrm{~d} \omega_{a s}, \\
& I_{n r}=\int_{-\infty}^{+\infty}\left|P_{n r}\left(\omega_{a s}\right)\right|^{2} \mathrm{~d} \omega_{a s} .
\end{aligned}
$$

It is the difference of resonant signal intensity integration and non-resonant background intensity integration. The optimization of this objective functional guarantees sufficiently large resonant signal when suppressing the background. With $\Delta=50 \mathrm{~cm}^{-1}$ and $\Gamma=4.8 \mathrm{~cm}^{-1}$, the numerical optimization result with respect to the phase functions of all the three pulses is shown in Figure 3 and the corresponding CARS spectrum is shown in Figure 4. From the simulation results, the optimal phase shaping configuration consists of a shaped probe pulse and unshaped pump and Stokes pulses, which suppresses the background to a small value across a broad frequency band in the spectrum, while the resonant signal remains large. The picture for the optimal phase profile tells that it is a quasi-linear, although it is not exact linear phase shaping. In addition, the amplitude profile of the shaped probe pulse in time domain also reveals a quasi-time-delay shape. In other words, the time delay scheme, a well-known method for the background suppression, is a quasi-optimal

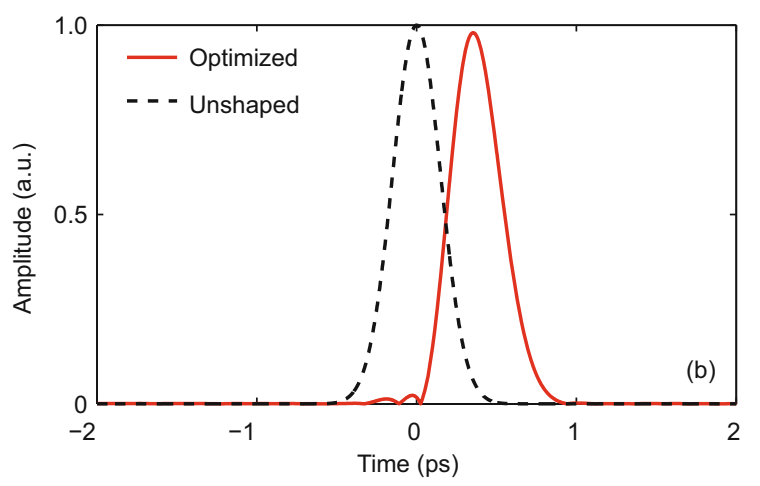

Figure 3 Optimal phase profile of the probe pulse (red solid lines) using numerical optimization in CMA-ES algorithm for the maximization of $I_{r}-I_{n r}$. (a) and (b) correspond to the frequency and time domains, respectively. The black dashed line in the right panel is the unshaped transform limited pulse. $\Delta=50 \mathrm{~cm}^{-1}$ and $\Gamma=4.8 \mathrm{~cm}^{-1}$. 


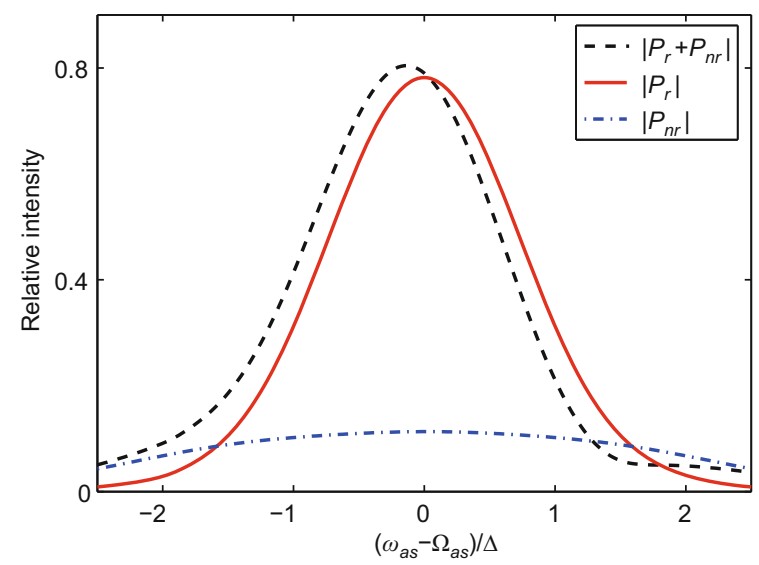

Figure 4 Spectrum of maximal $I_{r}-I_{n r}$. Here, the background (blue dash dotted line) is suppressed to be a small value across a broad frequency band, while the resonant signal (red solid line) remains large.

phase shaping scheme for the broadband background elimination, if the objective functional is defined as in eq. (18). For other types of objective functionals which place more importance on the broadband background elimination, it still holds that the time delay scheme is the quasi-optimal phase shaping scheme. Although the time delay scheme could not yield narrow-band spectrum, it has almost the best performance on the broadband non-resonant background suppression, which is especially advantageous when the background is very large. For practical considerations, appropriate combinations of the time delay scheme and other phase shaping schemes could achieve optimal performance for the signalbackground control in CARS.

\section{Conclusions and open questions}

This paper reviews the theory of optimal quantum measurements of finite-dimensional systems and CARS. Analytical and numerical results demonstrate effect of the corresponding strategies. For the measurement-induced control in finite-dimensional quantum systems, the necessary conditions for optimal measurements are obtained. Some analytical and numerical results are presented for different cases. In the CARS control, the optimal phase shaping scheme to achieve maximal resonant signal or minimal background at a specific frequency is simple, that is, only the probe pulse needs to be shaped while keeping pump and Stokes pulses in transform limited forms. As a balance of the resonant signal enhancement and non-resonant background suppression, the optimization of the objective functional $\left|P_{r}\right|^{2}-k\left|P_{n r}\right|^{2}$ could generate a CARS signal with large resonant signal and small background simultaneously, and an arctan-type optimal phase function is found for the probe pulse. Phase shaping schemes for broadband are investigated numerically, and it is found that the well-known time-delay scheme is a quasioptimal phase shaping scheme for the broadband background elimination. These control strategies could give us some hints toward designing new type quantum microscopes and quantum sensors.

Until now, the measurement-induced control is completely solved only for two-level quantum systems and several simple multi-level quantum systems, and the phase shaping technique is employed to improve the signal-to-background ratio in CARS when only one vibrational Raman mode is excited. However, real systems are much more complex. For example, they may be general multi-level systems, and more than one vibrational Raman modes are likely to be excited simultaneously by the incident lasers. By expanding the formulas to general multi-level systems and changing the corresponding control objectives, the selective excitation of Raman modes and maximal population transfer to a target state can be achieved. However, these complex cases often have no analytical solutions, and need to be further studied in the future.

Quantum measurement is an indirect control approach in the manipulation of quantum dynamics, while phase shaping of laser pulses is a direct control strategy. They can both be utilized to achieve control objectives of quantum systems. Phase shaping techniques are often employed in experiments to control the dynamics of quantum systems, and this could also be achieved by measurement-induced control in some cases. For example, we could control the excitation of quantum systems by pulse shaping, and then control the dynamics by both pulse shaping and measurement-induced control.

There are still many open questions to explore. The task of finding the state transfer probability between two general mixed states seems to be formidable. Do we have analytical solutions for some special mixed states? Can we solve this problem in single measurement case or find some new type of target functions to simplify the optimal procedure? In the CARS control, we only study pulse-shaping in time domain. As is known, the space resolution of CARS microscopy can not exceed the diffraction limit of the incident laser, without employing quantum effects. The quantum coherent control strategies by pulse-shaping in space domain to improve the space resolution of CARS microscopy have not been studied carefully up to now. Another point to be noticed is the chemical selectivity in CARS microscopy. Experiments have demonstrated that not only the CARS signals from different vibrational modes in one molecule but also from different molecules can interfere. By adjusting the pulse phases, the signal from one special molecule can be effectively enhanced while that from another type of molecules [41] is suppressed. Thus how to improve the chemical selectivity and space resolution of CARS microscopy simultaneously by pulse-shaping technique in time and space domain needs to be explored systematically in the future.

Gao was supported by Open Foundation of State Key Laboratory of Robotics (RLO201011), and Shuang would acknowledge the support from the National Natural Science Foundation of China (61074052 and 61072032), 
Foundation of the President of Hefei Institutes of Physical Science CAS and Open Foundation of State Key Laboratory of Precision Spectroscopy.

1 Dirac P A M. The Principles of Quantum Mechanics. London: Oxford University Press, 1947. 131-135

2 Von Neumann J. Mathematical Foundation of Quantum Mechanics. Princeton: Princeton University Press, 1955

3 Bohm D. A suggested interpretation of the quantum theory in terms of "hidden" variables I. Phys Rev, 1952, 85: 166-179

4 Bohm D. A suggested interpretation of the quantum theory in terms of "hidden" variables II. Phys Rev, 1952, 85: 180-193

5 Everett H. "Relative state" formulation of quantum mechanics. Rev Mod Phys, 1957, 29: 454-462

6 Zeh H D. On the interpretation of measurement in quantum theory. Found Phys, 1970, 1: 69-76

7 Zurek W H. Decoherence, einselection, and the quantum origins of the classical. Rev Mod Phys, 2003, 75: 715-775

8 Nielsen M A, Chuang I L. Quantum Computation and Quantum Information. London: Cambridge University Press, 2000

9 Balachandran A P, Roy S M. Quantum anti-zeno paradox. Phys Rev Lett, 2000, 84: 4019-4022

10 Pechen A, Il'in N, Shuang F, et al. Quantum control by von neumann measurements. Phys Rev A, 2006, 74: 052102

11 Shuang F, Zhou M, Pechen A, et al. Control of quantum dynamics by optimized measurements. Phys Rev A, 2008, 78: 063422

12 Shuang F, Pechen A, Ho T S, et al. Observation-assisted optimal control of quantum dynamics. J Chem Phys, 2007, 126: 134303

13 Maker P, Terhune R. Study of optical effects due to an induced polarization third order in the electric field strength. Phys Rev, 1965, 137: A801-A818

14 Duncan M, Reintjes J, Manuccia T. Scanning coherent anti-stokes raman microscope. Opt Lett, 1982, 7: 350-352

15 Cheng J, Volkmer A, Book L D, et al. An epi-detected coherent antistokes raman scattering (e-cars) microscope with high spectral resolution and high sensitivity. J Phys Chem B, 2001, 105: 1277-1280

16 Volkmer A, Cheng J, Xie X S. Vibrational imaging with high sensitivity via epidetected coherent anti-stokes raman scattering microscopy. Phys Rev Lett, 2001, 87: 023901

17 Cheng J, Xie X S. Coherent anti-stokes raman scattering microscopy: Instrumentation, theory, and applications. J Phys Chem B, 2004, 108: 827-840

18 Evans C L, Xie X S. Coherent anti-stokes raman scattering microscopy: Chemical imaging for biology and medicine. Ann Rev Anal Chem, 2008, 1: 883-909

19 Oron D, Dudovich N, Yelin D, et al. Narrow-band coherent antistokes raman signals from broad-band pulses. Phys Rev Lett, 2002, 88: 063004

20 Oron D, Dudovich N, Yelin D, et al. Quantum control of coherent antistokes raman processes. Phys Rev A, 2002, 65: 043408

21 Dudovich N, Oron D, Silberberg Y. Single-pulse coherently controlled nonlinear raman spectroscopy and microscopy. Nature, 2002, 418: 512 514

22 Oron D, Dudovich N, Silberberg Y. Femtosecond phase-andpolarization control for background-free coherent anti-stokes raman spectroscopy. Phys Rev Lett, 2003, 90: 213902

23 Zhu W, Rabitz H. Closed loop learning control to suppress the effects of quantum decoherence. J Chem Phys, 2003, 118: 6751-6757

24 Shuang F, Rabitz H. Cooperating or fighting with control noise in the optimal manipulation of quantum dynamics. J Chem Phys, 2004, 121: 9270-9278

25 Shuang F, Rabitz H. Cooperating or fighting with decoherence in the optimal control of quantum dynamics. J Chem Phys, 2006, 124: 154105

26 Shuang F, Rabitz H, Dykman M. Foundations for cooperating with control noise in the manipulation of quantum dynamics. Phys Rev E, 2007, 75: 021103

27 Pechen A, Rabitz H. Teaching the environment to control quantum systems. Phys Rev A, 2006, 73: 062102

28 Pechen A, Rabitz H. Incoherent quantum control. Qtm Prob Wht Noi, 2008, 23: 197-211

29 Judson R S, Rabitz H. Teaching lasers to control molecules. Phys Rev Lett, 1992, 68: 1500-1503

30 Roa L, Delgado A, Ladrón de Guevara M L, et al. Measurement-driven quantum evolution. Phys Rev A, 2006, 73: 012322

31 Vilela Mendes R, Man'ko, V I. Quantum control and the strocchi map. Phys Rev A, 2003, 67: 053404

32 Sugny D, Kontz C. Optimal control of a three-level quantum system by laser fields plus von neumann measurements. Phys Rev A, 2008, 77: 063420

33 Zhang M, Dai H Y, Xi Z, et al. Combating dephasing decoherence by periodically performing tracking control and projective measurement. Phys Rev A, 2007, 76: 042335

34 Dong D, Lamb J, Petersena I R. Robust incoherent control of qubit systems via switching and optimisation. Int J Control, 2010, 83: 206-217

35 Sugawara M. Quantum dynamics driven by continuous laser fields under measurements: Towards measurement-assisted quantum dynamics control. J Chem Phys, 2005, 123: 204115

36 Sugawara M. Measurement-assisted quantum dynamics control of 5level system using intense cw-laser fields. Chem Phys Lett, 2006, 428: 457-460

37 Wang Y, Wu R, Chen X, et al. Quantum state transformation by optimal projective measurements. J Math Chem, 2011, 49: 507-519

38 Ostermeier A, Gawelczyk A, Hansen N. A derandomized approach to self-adaptation of evolution strategies. Evol Comput, 1994, 2: 369-380

39 Hansen N. The cma evolution strategy: A comparing review. Stud Fuzz Soft Comp, 2006, 192: 75-102

40 Gao F, Shuang F, Shi J, et al. Optimal coherent control of cars: Signal enhancement and background elimination. arXiv: 1109.6704v3

41 Konradi J, Singh A K, Matemy A. Selective excitation of molecular modes in a mixture by optimal control of electronically non-resonnant femtosecond four-wave mixing spectroscopy. J Photoch Photobio A, 2006, 180: 289-299

Open Access This article is distributed under the terms of the Creative Commons Attribution License which permits any use, distribution, and reproduction in any medium, provided the original author(s) and source are credited. 